\title{
Determinant of treatment delay in the first episode of psychosis: a qualitative study
}

\begin{abstract}
Aims: The aims of this study were to investigate patterns of requesting treatment amongst first-time user psychotic patients and to reveal the causes for delay in terms of accessing psychiatric care.
\end{abstract}

Methods: Qualitative semi-structured interviews were carried out with 25 family relatives of people with schizophrenia. Thematic analysis was used to analyse the data. The thematic analysis highlighted four themes: lack of awareness about mental illness the symptoms associated, perceived stigma, the role of extended family, and financial problems.

Results: The findings revealed that perceived stigma and fears about being labelled are the main barriers to requesting early psychiatric healthcare. Furthermore, the participants cited many different reasons linked to getting help, for example, lack of knowledge about mental illness and its related symptoms which lead to a delay in seeking help at the early stages of the illness, family and financial factors. It has been thus argued there is a need for mental health awareness campaigns to be conducted within communities.

Conclusions: The study highlights the importance of enhancing knowledge about schizophrenia related spectrum to ensure patients seek treatment in a timelier manner.

Keywords: duration of untreated psychosis, first-episode psychosis
Volume 9 Issue $3-2018$

\author{
Abd Alhadi Hasan \\ Fakeeh College for Medical Sciences, Saudi Arabia
}

Correspondence: Abd Alhadi Hasan, Fakeeh College for Medical Sciences, Alhamra district, Palestine street, Jeddah, Saudi Arabia,Tel 0785278005,Email abdhasan2007@yahoo.com

Received: January 29, 2017| Published: May 07, 2018

\section{Introduction}

When an individual first presents with mental illness, the symptoms are not very noticeable to people who are do not have knowledge about them. This means there is usually a delay in patients accessing treatment. ${ }^{1}$ Seeking treatment is an continuing process in which symptoms of an illness mature ${ }^{2}$ and are progressively observed and assessed. They are then eventually treated through psychiatric treatment. ${ }^{3}$ The patient and their family acquire their own perception about how effective a treatment is based on changes they observe in behaviour and mental state. They then use this experience to base their future treatment decisions on. ${ }^{2}$ When a patient experiences their first episode of psychosis, the psychosis begins. ${ }^{4}$ The process of gaining treatment is continual until they have recovered. The time period between the first psychotic episode gaining psychopharmacological treatment is referred to as the duration of untreated psychosis (DUP), and this is usually delayed. ${ }^{5}$ Getting immediate treatment after experiencing psychotic symptoms is a vital factor in a positive treatment outcome. ${ }^{6}$ It has been revealed that the longer a person untreated for their psychotic symptoms, the less chance they have of recovery and the more resistant to treatment they are, therefore there is a higher risk of relapse and a poorer overall prognosis. ${ }^{1,7}$ Furthermore, Marshall et al..$^{5}$ revealed that a longer DUP is associated with poorer treatment outcomes, however a shorter DUP leads to a better treatment outcome. A community based study was carried out in the UK with first episode psychosis patients. It was found that social factors such as family involvement is vital in terms of reducing DUP. Thus a result, the researchers argued social factors are instrumental in terms of patients seeking initial and continuous psychiatric treatment and care. ${ }^{7}$ Another study proposed a large population community based program "early Treatment and Intervention in Psychosis (TIPS)" aimed at the general population to increase their awareness about mental through local media. It was found that the program was effective in terms of decreasing the median of DUP. ${ }^{8}$ The current literature about treatment seeking behaviour amongst psychiatric patient suggests that seeking treatment when psychiatric disorders first begin is determined by various factors. ${ }^{9}$ These factors are early age of onset, the type of diagnosis, family and society beliefs about mental illness, worries about being labeled and the level of stigma perception. Based on the notion of seeking treatment early, Coton $\mathrm{X}^{10}$ revealed that Asian population, namely people from Cambodia, lack knowledge about the symptoms of mental illness and this caused them to delay treatment seeking. This has also been argued by Chong et al. ${ }^{11}$ who informed that attitudes and opinions about supernatural causes of mental illness also have an effect on the patient's chosen care plan.

There are also other factors related to people who experience psychosis symptoms to request early treatment, such as strength of family bond, family involvement in the treatment process, past experience of psychotic symptoms, in addition to experiencing positive symptoms of psychosis and aggressive behavior. ${ }^{12}$ Aggressive behavior can occurs on a personal level, while a society's views about mental illness are categorized at community level. In terms of other forms of mental illness, PDwS experience stigma, especially in developing countries. ${ }^{13}$ This can lead to families and their ill relative to concealing the problem and getting treatment from non-medical health professionals. Marthoenis $\mathrm{M}$ et al. ${ }^{13}$ revealed that a lack of awareness and/or difficulty in accessing mental health services in developing countries means people look for help from unconventional services, including traditional medicine, general practitioners and 
physician rather than psychiatrist. The majority of studies in this field of research have excluded mental illness. As a result, the current study aims to investigate the reasons for delay in seeking psychiatric help.

\section{Methods}

This study will use a qualitative approach with semi-structured interviews as this allows the researcher to investigate the reasons for delay in seeking psychiatric help amongst patients with schizophrenia in an in-depth manner. Participants were enlisted from four outpatient mental health clinics in Amman, Jordan. A purposeful sampling was used to sample the participants to participate in the semi-structured interviews. Inclusion criteria were that the participants must have been diagnosed with schizophrenia according to DSM-V criteria. In addition, they had to be able to speak either Arabic or English. Ethical approval was gained from the Medical Research Ethics Committee from the Ministry of Health in Jordan. The researcher developed a series of topics for discussion to guide the interviews process to ensure as much in-depth information was collected as possible. The topic guide was developed based on the aims of the study aims to determine the factors behind delays in getting help from psychiatric services. The interviews were recorded and then and transcribed verbatim. Each interview transcript was appraised by two independent raters to ensure credibility. Both raters were studying a graduate degree in mental health nursing. The data were analysed using thematic analysis to produce themes. Data redundancy ensued when no new information arose from the interviews. There was no time limit set for the interviews, however on average; each interview lasted for around 45-60 minutes. Before ach interview began, the participants were informed they were able to ask questions and were able to refuse to answer any of the questions they were asked. Furthermore, the participants were informed they had the right to withdraw from the interviews at any time and did not have to give a reason for doing so. The interviews were recorded to allow the interviewer to focus on the questions and answers and be more interactive with the participants. This allowed the interviewer to ask follow up questions about issues raised. Recording the interviews also allowed the researcher to avoid marking errors with note taking. All of the interviews were conducted in Arabic, and transcribed in Arabic, but were then translated into English for analysis. The transcripts were then back-translated into Arabic to ensure there was no loss of meaning in what the participants had stated and to authenticate conceptual equivalence of translation [38]. The English versions of the transcripts were checked by primary investigator (PI) and Research assistant (RS). PI carried out the initial coding of all interviews and RS conducted the initial coding for a random selection of interviews then the codes were compared.

\section{Data analysis}

All of the data were analysed using inductive thematic analysis, which was done in six stages ${ }^{14}$ as detailed below.

1. The iinterview transcripts were read and re-read over and over again to ensure a deep understanding was gained of the participants' statements. ${ }^{14}$

2. The initial codes were developed; a complete coding approach was used in this to highlight everything within the data set which may have been relevant to the research aims..$^{14,15}$

3. All of the similar codes gathered together into possible themes A preexisting coding frame was not used. Instead, appropriate excerpts from the data set were collated to develop themes. ${ }^{14,15}$

4. Possible themes were reviewed and a thematic map was developed.

5. The identified themes and subthemes were cross-checked and checked against the dataset to make sure they were comprehensible, distinctive, and consistent and worked together.

6. Themes were reflected an the semantic level of data with illustrative quotes from participants being selected. ${ }^{14}$

\section{Results}

\section{Lack of Awareness about mental illness the associated symptoms}

This theme includes the participants' views about the behaviour of their ill relative who was experiencing early psychosis. Overall, there was a lack of knowledge about schizophrenia as over two thirds of the participants stated the reason behind delayed access to treatment was because of low levels of knowledge amongst their family members about mental illnesses. Most of the participants believed mental illness was caused by supernatural episodes, for example, black magic, ghosts, or being possessed. Therefore they would try to treat their relative though using folk exorcism to banish the evil spirits.

“..... Previously we thought that this illness [schizophrenia] resulted from the possession of devils inside his body which asked him to perform these behaviours. Also, we \{ah\} thought this illness stemmed from being nervous, stressed or some unknown fear....." (P7, Male)

On the same line of though, one participant believed schizophrenia might be caused by of charm, and would ask a magician to get rid of it:

"..... In the beginning when he started to behave abnormally or say absurd words, I thought someone had cursed him because he was resourceful and active [....]. We went to many people for help but it was no benefit to us....." (P15, Female)

The lack of knowledge about schizophrenia was also revealed by the participants in that over two thirds of them thought symptoms of mental illness were linked to somatic illness. For example:

“.... when I informed my family that I heard voices $\{. .$.$] , they took$ me to general physician...."(P11, Female)

Most of the participants also though schizophrenia is a split personality.

“.... I thought that my illness was considered as many personalities inside my body [...] especially when I heard voices....." (P22, Male)

“.... I also thought schizophrenia has split $\{a h . .$.$\} my personality$ in two parts...." (P23, Female)

The majority of the participants informed that they requested treatment from a traditional healer

"..... because we did not know sign and symptoms of mental illness.... My father asked a religious person to help me....." (P5, Male)

The excerpt below suggests that, in terms of the nature of their diagnosis, the participants were affected by cultural factors regardless of their level of education: 
"As I am his wife and in our culture we should tolerate their behaviours.... When I went to his family complaining him they were asked me to wait because he is nervous person...." (P10, Female)

Moreover, most of the participants used traditional healers for the first treatment, but they did not provide them correct information about the illness. On many cases, apart from being provided with incorrect information about mental illness, the traditional healers also prevented them from getting treatment from the psychiatric clinic.

".....We went to see a magician and he said that he expelled their souls out of my mind.... He told us that there was no need to visit mental health service as medication damages body..." (P20, Male)

The type of psychotic symptoms also played a vital role in pursuing treatment. For example, some of the participants stated that when the positive symptoms of schizophrenia were very noticeable, their relative immediately accessed a mental health service.

"When she started to Speak with herself and was getting worse, unpredictable onset of this episode, might hit anyone. ... (P3, Male)

"...He hit his father few days ago, then my family believed that I have mental health problem.... That a reason why did they bring me to mental clinic" (P23, Male)

Conversely, patients with had mainly negative symptoms were less likely to be deemed as having a mental illness and therefore this caused delays in them pursuing treatment.

“..... I preferred to be alone all the times.... My family thought this was related to my personality characteristics..." (P18, Male)

"..... My family was not assured if some symptoms which I had related to the mental illness, they thought..." (P11, Male)

"....It was a normal behavior, I was lazy, that was why they did not take me to mental health hospital ..." (P7, Male)

Other people would also perceive the person's illness to be something different to what It was. For example, one mother strongly believed that her son's symptoms were caused by a seizure and other nervous problems, because she had been made to believe this by her friends.

Inappropriate information about patients' behavior was also obtained from others. A mother, for instance, was convinced by her friends that her son symptoms were related to the seizure and other nervous system problem.

“...... When someone is smiling or talking to himself like he (the patient) is, people said that is nerve disorder....." (P10, Female)

In addition to inclination to pursue treatment from traditional or faith healers, many of the participants took their ill relative to a neurologist, as they believed the mental illness was due to a disorder in a part of the brain.

“..... We usually went to nerve specialist to treat me but all of medications were useless...." (P25, Female)

In sum, it can be argued the participants had a distinct lack of understanding about mental illness and this is one of the main reasons for the delay in pursuing psychiatric treatment.

\section{Perceived stigma}

The theme of perceived stigma concerns the beliefs the participants had about society's negative reaction to how their increasingly ill relative behaved. In general, the participants stated society's view about mental illness was very negative.

“.... When my son walks in the street... some children follow him and shout crazy crazy... this is very harsh attitude for mentally ill people...." (P14, Male)

"..... Our society makes mental ill people as a joke and they laugh at them... we look at antipsychotic medication inferiorly..." (P10, Female)

The perceived stigma of having a relative with a mental illness is very common amongst Jordanian people. The extent of the stigma does however vary amongst families. Some families felt very stigmatized and tried to hide their relatives from their neighbor.

"....We changed our home many times to be away from our neighbors, particularly when his illness being known to others..." (P7, Male)

"I felt alone when several of my friend decided to disconnect her relationships with me after my husband aggressive time.... as a result, we are being discriminated by other people in our district and we moved home.."

“..... Moreover, embarrassment and guilt because of her mental illness urged us to minimise our contact with others in order to reduce the number of people who knew about her illness....." (P3, Male)

“.... The negative view in the community has impacted on our family; our neighbourhood know he has an illness, but they do not know details about his illness including the (diagnosis) [....]. Some of the surrounding people changed their views about us....." (P23, Female)

Dissimilar to the previous statement, other participants perceived mental illness to be governed by God, who can provide a cure. Subsequently, they held a positive opinions about mental illness and had a very minimal perceived stigma.

"We believe everything in this life is predetermined from Allah.... We have to tolerate this and good reward will be afforded for us... ."(P19, Female $)$

" I look for mental illness as other form of illness such as hypertension which can be controlled by medication and the life remains positive...." $(\boldsymbol{P} 1$, Male $)$

From the views of the participants it seems stigma has a very negative effect on family integrity. Therefore most of the participants, namely the males informed of a feeling od detachment and stated they had stressful relationships with their relatives. This is revealed in the statements made regarding the perceived stigma on their interpersonal relationships.

“.... My illness caused me to lose my job [.....]. My family, especially my parents blamed me and said I pretended to be lazy and tired just because I did not like to work. This enforced me to leave our house and live alone....." (P2, Female)

“..... My wife decided to leave me when she felt discriminated by others and her response became defensive and shy...." (P7, Male) 
Further analysis of above comments revealed that there might be another reason to delayed accessing of treatment, which is the stigma associated with mental illness and the fear of being labeled when receiving a diagnosis.

\section{The role of extended family}

It has been found family members play a key role in person's with mental illness accessing treatment from a psychiatric clinic because all family members needed to agree to this before taking action. The oldest member of the family makes the final decision about when is the right time to get treatment. The excerpt below reveals how one male's treatment was delayed because his father finally allowed him to seek treatment at a hospital, after he had been suffering with symptoms for a long period of time.

“... I read about my illness symptoms and it appeared to be a mental illness. So I wanted to visit doctor a long time ago, but my father never allowed me to do so. Now the my father is fed up as her behavior is getting worse, so now she allowed us to. . so he agreed to take him there...." (P17, Male $)$

Other participants also referred to the fact that their family had prevented them from pursuing treatment at a psychiatric clinic. One participant informed he was very frustrated because it took him a long time to convince his family that he needed treatment. Furthermore, many of the participants gave negative comments regarding the mental health services available in Jordan and stated they have problems gaining information.

"...You know, we asked many local people about these symptoms which I had as hearing voices or seeing views around me, but we could not reach a definitive answer....."(P19, Female)

"... We did not have any information about governmental psychiatric clinic... accidently, my father friend informed him about location the nearest clinic.. we called them and booked an appointment..."(P2, Female)

Overall, the statements made by the participants showed the family has a significant impact on delaying access to treatment from a psychiatric clinic and usually had to wait a long time for their family to agree to this.

\section{Financial problems}

The majority of the participants indicated financing causing them to delay access to treatment. Some would ask for money or borrow it from relatives to pay for treatment.

".... We heard mental treatment is available in private clinic which costs people a lot.... I asked a job owner for some money to pay for treatment...."(P7, Male $)$

".... Antipsychotic medication is very expensive as well as lab test... so we decided to use less expensive method..."(P19, Female)

Furthermore, some of the participants stated they were aware that psychiatric clinic has to process official documents before they can be treated and many clinics ask patients to pay for their own treatment.

“... In a governmental clinic that I visited last month, it was free of charge. But they asked me to buy medication from private pharmacy which is very expensive...." (P20, Male)

It is noteworthy that participants appeared financial restrain would change family hope in getting recovered from illness" (P23,Female)

\section{Discussion}

To the best of the researcher's knowledge, this is the first study that has been carried out in an Arab country that has investigated the factors that cause a delay in DUP seeking treating from a psychiatric clinic. The treatment of mental illness in Jordan does not normally start as soon as a person starts to experience positive or negative symptoms of schizophrenia. In the beginning, most people think the symptoms of mental illness are part of normal behaviour. In a general sense, a lack of awareness about mental illness and its symptoms usually cause a delay in getting treatment.

In addition to knowledge about mental illness is also, people in Jordan also feel a sense of shame as well as helpless and hopeless and do not feel empowered to get help. This concurs with the findings of Lysaker et al. ${ }^{16}$ and Sibitz I et al. ${ }^{17}$ who have informed that a lack of awareness about schizophrenia is related to stigma and stereotypes about DUP and this can result in depression. Similarly, Pickett et al. ${ }^{18}$ and Alegría et al. ${ }^{19}$ also discovered that low levels of knowledge about mental illness are associated with a lack of empowerment to get treatment which leads to the patient experiencing a negative psychological effect and social stigma and that individuals would use negative coping strategies to cope with their illness, such as secrecy and avoidance. Many of the participants in this study also believed that supernatural causes are to blame for mental illness and therefore family members would encourage their ill relative to seek help from faith or traditional healers. This is common amongst people in developing countries, for example it also occurs in Singapore. Getting help from faith and traditional healers is common and this prevents individuals from seeking treatment from health professionals, thus individuals remain untreated. Therefore, it is vital that family members of mentally ill individuals are taught about mental illness so they seek the correct path of treatment in the beginning.

In Jordan, the beliefs about the aetiology of mental illness vary. There are some who think schizophrenia is a weakness of character, the person is being lazy, or they are being punished for what their ancestors have done in the past. ${ }^{20-22}$ Others think that mental illness is a result of evil spirits, magic, a blow to the head, bad genes, emotional trauma, a punishment from God, or because of the evil eye. ${ }^{23,24}$ These beliefs about mental illness might bring about stigma because some think the individual is being punished for not having faith in $\mathrm{God}^{25}$ or for their $\operatorname{sins}^{26}$ which more than likely contributes to the stigma associated with mental illness in Arab countries. ${ }^{27}$ The perception of stigma prevents people from seeking help for mental illness and also from adhering to medication. ${ }^{17,28}$ What is more, in Arab cultures there is not much of a distinction between physical and psychological health (as there is in Western countries). Literature from Arab countries has informed that Arab-Muslims do not make a distinction between emotional and psychological distress from physical illness and most people somatise their illness, whereby those who are mentally ill present with an emotional disorder through physical symptoms. ${ }^{23,29,30}$ The difficulty in distinguishing between illnesses is further exacerbated by the individual's ability to cope with their symptoms, as well as the level of tolerance of family member's towards their behavioural issues. As a result, individuals tend to seek treatment from unconventional sources and do not consult healthcare professionals. ${ }^{25}$ One reason for this is because it is believed there is less of stigma attached to 
unconventional treatments. ${ }^{32}$ Studies by Gearing et al. ${ }^{31}$ and ${ }^{25}$ found that opinions and beliefs about mental illness are the main barriers for individuals in Middle Eastern-Arab countries accessing professional mental health treatment, or taking part in psychosocial treatments and service implementation. This is also in line with other studies conducted in Arab-Muslim countries, which have reported that when people do not understand mental illness, this increases the stigma and they are more likely to tolerate the symptoms of their mental illness without seeking professional help. ${ }^{32,33}$ There are also other studies that agree with this which have found that Asian families usually consult faith healers or take herbal supplements before seeking help from a psychiatric clinic. ${ }^{34,35}$ Families and significant others play an vital role in the treatment of people with mental illness in Arab countries. For example Boydell et al. ${ }^{36}$ conducted a qualitative study and found that many individuals with mental illness spoke of the key role their relative played in them seeking treatment for psychotic symptoms. In line with this, another qualitative study that was done in Jordan with DUP revealed that improving the knowledge of primary careers for individuals with schizophrenia had a positive effect on the treatment process and the time span of seeking treatment. In the current study, it may be that the role of the family and the fear of being labeled are connected. This current study took on the assumption that the extended family prevents those who are mentally ill from seeking treatment through fear of social stigma and being labeled. Families tend to keep the illness a secret because they fear rejection from others. What is more, people in Jordan tend to perceive being treated by a neurologist as preferable to being treated by a psychiatrist. This is because it is more expensive to be treated by a psychiatrist and there are also fewer stigmas associated with it. . $^{37,38}$

\section{Conclusion}

This study has revealed there are various factors that lead to Jordanians who have schizophrenia seeking psychiatric treatment, for example, incorrect knowledge and information about mental illness and its symptoms, stigma and financial barriers.

\section{Acknowledgment}

None.

\section{Conflict of interest}

The author declares that there is no conflict of interest.

\section{References}

1. Sheitman BB, Lieberman JA. The natural history and pathophysiology of treatment resistant schizophrenia. J Psychiatr Res. 1998;32(3-4):143-150.

2. Yang YK, Hsieh $\mathrm{HH}$, Wu $\mathrm{AC}$, et al. Help-seeking behaviors in relatives of schizophrenics in Taiwan. General Hospital Psychiatry. 2009;21(4):303-309.

3. Bou-Yong Rhi, Kyoo-Seob Ha, Yong-Sik Kim, et al. The health care seeking behavior of schizophrenic patients in 6 East Asian areas. International Journal of Social Psychiatry. 2005;41(3):190-209.

4. Michael T Compton. The First Episode of Psychosis: AGuide for Patients andTheir Families. $1^{\text {st }}$ ed. Oxford University Press:USA. 2009;288.

5. Marshall M, Lewis S, Lockwood A. Association between duration of untreated psychosis and outcome in cohorts of first-episode patients. Arch Gen Psychiatry. 2005;62(9):975-83.

6. Perkins DO, Gu H, Boteva K. Relationship between duration of untreated psychosis and outcome in first-episode schizophrenia: a critical review and metaanalysis. Am J Psychiatry. 2005;162(10):1785-804.

7. Morgan C, Abdul-A1 R, Lappin JM. Clinical and social determinants of duration of untreated psychosis in the $Æ S O P$ first-episode psychosis study. Br J Psychiatry. 2006;189:446-52.

8. Melle I, Larsen TK, Haahr U. Reducing the duration of untreated firstepisode psychosis: effects on clinical presentation. Arch Gen Psychiatry. 2004;16(2):143-50.

9. Bechard-Evans L, Schmitz N, Abadi S. Determinants of help-seeking and system related components of delay in the treatment of first-episode psychosis. Schizophrenia Research. 2007;96(1-3):206-214.

10. Coton X, Poly S, Hoyois P. The healthcare-seeking behaviour of schizophrenic patients in Cambodia. Int J Soc Psychiatry. 2008;54(4):328337.

11. Chong SA, Mythily, Lum. Determinants of duration of untreated psychosis and the pathway to care in Singapore. Int J Soc Psychiatry. 2005;51(1):55-62.

12. Goulding SM, Leiner AS, Thompson NJ. Family strengths: a potential determinant of the duration of untreated psychosis among hospitalized African-American first-episode patients. Early Interv Psychiatry. 2008;2(3):162-168.

13. Marthoenis M, Aichberger MC, Schouler-Ocak M. Patterns and Determinants of Treatment Seeking among Previously Untreated Psychotic Patients in Aceh Province, Indonesia: A Qualitative Study. Scientifica (Cairo). 2016: 9136079.

14. Virginia Braun, Victoria Clarke. Using thematic analysis in psychology. Qualitative research in psychology. 2008;3(2):77-101.

15. Green J, Thorogood N. Qualitative methods for health research. Sage. 2013.

16. Lysaker PH, Buck KD, Hammoud K. Associations of symptoms, psychosocial function and hope with qualities of self-experience in schizophrenia: Comparisons of objective and subjective indicators of health. Schizophr Res. 2006;82(2-3):241-249.

17. Sibitz I, Amering M, Unger A. The impact of the social network, stigma and empowerment on the quality of life in patients with schizophrenia. Eur Psychiatry. 2011;26(1):28-33.

18. Pickett SA, Diehl SM, Steigman PJ, et al. Consumer empowerment and self-advocacy outcomes in a randomized study of peer-led education. Community mental health journal. 2012;48(4):420-430.

19. Alegría M1, Polo A, Gao S. Evaluation of a patient activation and empowerment intervention in mental health care. Medical care. 2008;46(3):247-256.

20. Al-Krenawi A, Graham JR, Al-Bedah E. Cross-national comparison of Middle Eastern university students: Help-seeking behaviors, attitudes toward helping professionals, and cultural beliefs about mental health problems. Community Ment Health J. 2009;45(1):26-36.

21. Amal Hassan Fadlalla. Modest women, deceptive jinn: Identity, alterity, and disease in Eastern Sudan. Identities: Global Studies in Culture and Power. 2004;12(2):143-174.

22. Lam, CS, Tsang H, Chan F, et al. Chinese and American perspectives on stigma. Rehabilitation Education. 20(4):269-279.

23. Endrawes G, O’Brien L, Wilkes L. Mental illness and Egyptian families. Int J Ment Health Nurs. 2007;16(3):178-187.

24. Gearing RE, MacKenzie MJ, Ibrahim RW. Stigma and mental health treatment of adolescents with depression in Jordan. Community Ment Health J. 2015;51(1):111-117. 
25. Nasser Aloud, Amena Rathur. Factors affecting attitudes toward seeking and using formal mental health and psychological services among Arab Muslim populations. Journal of Muslim Mental Health. 2009;4(2):79-103.

26. Weatherhead S, Daiches A. Muslim views on mental health and psychotherapy. Psychology and Psychotherapy: Theory, Research and Practice. 2010;83(1):75-89.

27. Abdullah T, Brown TL. Mental illness stigma and ethnocultural beliefs, values, and norms: An integrative review. Clin Psychol Rev. 2011;31(6):934-948.

28. Fung KM, Tsang HW, Cheung WM. Randomized controlled trial of the self-stigma reduction program among individuals with schizophrenia. Psychiatry Res. 2011;189(2):208-214.

29. Alean Al-Krenawi, John R Graham, Jamil Kandah. Gendered utilization differences of mental health services in Jordan. Community Mental Health Journal. 2000;36(5):501-511.

30. Joshua Fogel, Daniel E Ford. Stigma beliefs of Asian Americans with depression in an internet sample. Canadian Journal of Psychiatry. 2005;50(8):470-478

31. Gearing RE, Schwalbe CS, MacKenzie MJ. Adaptation and translation of mental health interventions in Middle Eastern Arab countries: A systematic review of barriers to and strategies for effective treatment implementation. Int J Soc Psychiatry. 2013;59(7): 671-681.
32. Al-Makhamreh, Sahar, Hasna. Localising social work: Lessons learnt from a community based intervention amongst the Bedouin in Jordan. Social Work Education. 2012;31(8):962-972.

33. Okasha A. Mental health in the Middle East: an Egyptian perspective Clinical psychology review. 1999;19(8):917-933.

34. Rammohan A, Rao K, Subbakrishna D. Religious coping and psychological wellbeing in carers of relatives with schizophrenia. Acta Psychiatrica Scandinavica. 2002;105(5):356-362.

35. Huang, Hung BJ, Sun F, et al. The experiences of carers in Taiwanese culture who have long-term schizophrenia in their families: a phenomenological study. J Psychiatr Ment Health Nurs. 2009;16(10):874-883.

36. Boydell KM, Gladstone BM, Volpe T. Understanding help seeking delay in the prodrome to first episode psychosis: a secondary analysis of the perspectives of young people. Psychiatr Rehabil J. 2006;30(1):54-60.

37. Al-Adawi S, Dorvlo AS, Al-Ismaily SS, et al. Perception of and attitude towards mental illness in Oman. Int J Soc Psychiatry. 2002;48(4):305-317.

38. Successful qualitative research: A practical guide for beginners. Sage. 2013 